\title{
The effect of bermuda grass root morphology on the displacement of slope
}

\author{
Xiao-Lei JI, ${ }^{1}$ Ping YANG, ${ }^{2}$ \\ ${ }^{1}$ College of Construction Engineering, Jiangsu Open University, Nanjing, Jiangsu 210019, China; \\ ${ }^{2}$ College of Civil Engineering, Nanjing Forestry University, Nanjing, Jiangsu 210037, China
}

\begin{abstract}
During recent years, with the drastic development of highway, lots of slopes are produced which cause the increase of geological disasters including soil erosion on the slope and shallow sliding surface, it makes significant impact on the ecological environment. Adopting ecological protection technique of plant slope protection not only can prevent geological disasters, but also benefit the protection of ecological environment. In the paper, bermuda grass, as one kind of ordinary slope protection plants, was chosen as specimen, and the root-soil composite was regarded as organic system which consists of soil and root system; the effect of root morphology on displacement of slope surface under the function of rain erosion and slope runoff erosion was studied and analyzed by using nonlinearity finite calculation method. The result indicates Bermuda grass root system can defense the rain erosion of slope surface soil, restrain the soil displacement and prevent water and soil erosion of slope.
\end{abstract}

\section{Introduction}

Traditional slope protection generally use geotextiles protection measures such as bolt shotcrete or dry pitching, but these measures totally confine the growth environment of plant while protecting the slope[1-3], this makes the original plant on the slope unable torecover in short period of time and destroy the ecological environment[4-7], besides, geotechnical structure always produce weathering and shedding over time which induce the decrease of strength, so the performance of protection becomes worse and worse. The ecological protection technique of plant slope protection not only can benefit the slope stability, but also can be good to the ecological environment preservation [8-10], so it is applied to gradually replace the traditional technique to protect slope and conduct greening[11-13]. In the paper, the bermuda grass root system was used as slope protection plant, the engineering simulation finite element software, ABAQUS, was applied to conduct digital analysis, this software can be used to work out problems range from comparatively easy linear analysis to complicated nonlinear analysis, meanwhile, it has abundant unit database that can simulate any geometrical shape so as to tackle the issues about strain and displacement of slope. The effect of root morphology on displacement of slope surface under the function of rain erosion and slope runoff erosion was studied and analyzed by using ABAQUS, this makes it feasible to quantitively analyze soil solidity of root system and provide the effective proof for the plant choice of ecological slope protection[14-18].

\section{Numerical model size and parameters}

\section{1 slope model and bounding condition}

The model was simplified to be convenient for calculation and the slope soil was assumed to be same and isotropic, the model of slope is showed in Fig. 1 and Fig.2. The bounding condition of this model is that the left and right lateral boundaries constrain the displacement in $\mathrm{x}$ direction when the bottom boundary

Corresponding author: E-mail:jixlsuny@126.com 
constrains the displacement in $\mathrm{x}$ and $\mathrm{y}$ direction. The

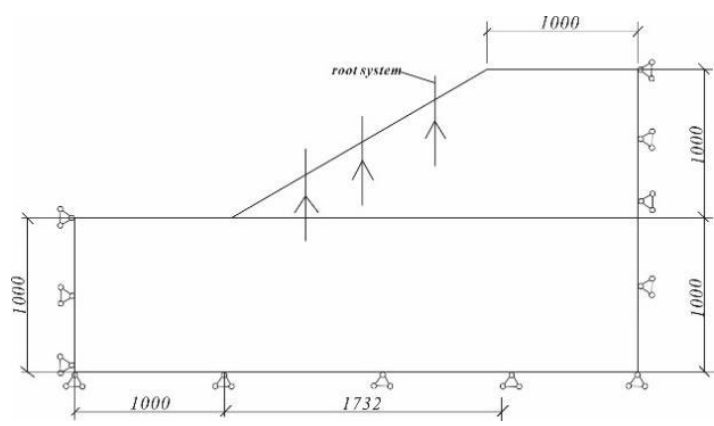

Fig.1 Diagram of slope physical parameters of slope material are listed in Tab.1.

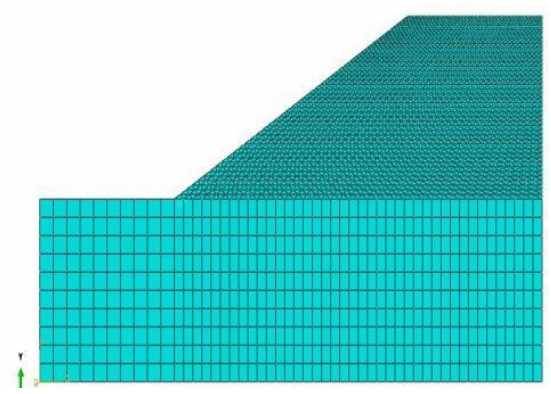

Fig.2 Finite element grid diagram of slope

Table.1 Parameters of soil

\begin{tabular}{|c|c|c|c|c|c|c|}
\hline Item & $\begin{array}{c}\text { gravity } \\
\left(\mathrm{kg} / \mathrm{m}^{3}\right)\end{array}$ & $\begin{array}{c}\text { Internal fraction } \\
\text { angle } \varphi\left({ }^{\circ}\right)\end{array}$ & $\begin{array}{c}\text { Moisture content } \\
(\%)\end{array}$ & $\begin{array}{c}\text { Elastic Modulus } \\
(\mathrm{Mpa})\end{array}$ & $\begin{array}{c}\text { Poisson } \\
\text { ratio }\end{array}$ & $\begin{array}{c}\text { Cohesive } \\
\text { force }(\mathrm{kPa})\end{array}$ \\
\hline Value & 18 & 15.2 & 27.7 & 4 & 0.3 & 7.12 \\
\hline
\end{tabular}

\section{2 model of root system}

The bermuda grass root system was simplified in numerical calculation, the main root and fibrous root are treated in equal diameter, the model of root system is ideal elastic model of flexible pole element which can only sustain tensile force but the press force. The diameters of main root and fibrous are $5 \mathrm{~mm}$, their length was considered as $200 \mathrm{~mm}, 300 \mathrm{~mm}, 400 \mathrm{~mm}$, the respective model is showed in Fig.3.
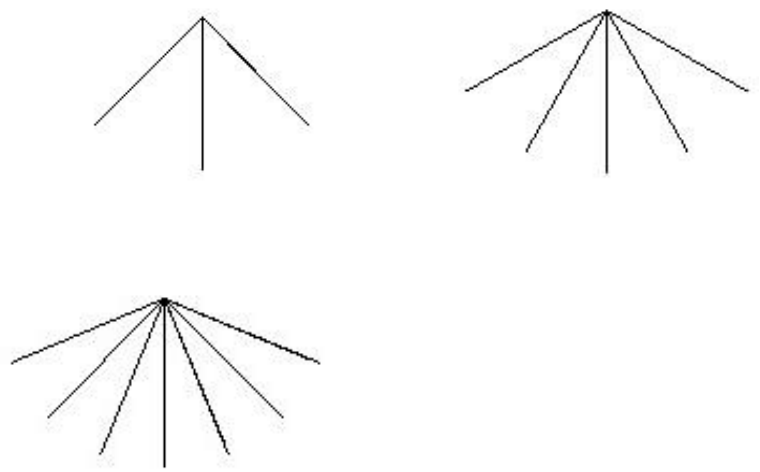

Fig.3 Schematic diagram of root distribution

\section{3 model of root-soil composite}

The root-soil composite was regarded as an integrate in the calculation without considering the sliding between soil and root. The root system and soil were set as materials with different parameters. It was assumed that the soil and root system connected with each other directly and the displacement coordination was realized automatically. In the model, the root system was adopted as liner structure because the root system is regarded as pole element. The interface between soil and root system was tackled with Embedded region in the bounding constraints order in ABAQUS, the root system was set as Embedded Region embedded into surrounding soil.

\section{Result and analysis}

In the condition that the diameter of rain point is $5 \mathrm{~mm}$, the flow speed of rain point is $9.11 \mathrm{~m} / \mathrm{s}$, the flow depth of surface water is $100 \mathrm{~mm}$, the effect of different arrangement density, different length, and different amount of fibrous root of root system on the horizontal and vertical and total displacement of slope surface was investigated. The finite element displacement filed cloud map without implanting root system is showed in Fig.4, the finite element displacement field cloud map with implanting root system is showed in Fig.5, by comparing these two figures, it was indicated that the displacement of slope with root system decreased obviously, the special calculation results are listed in Tab.2 and Tab.3. 


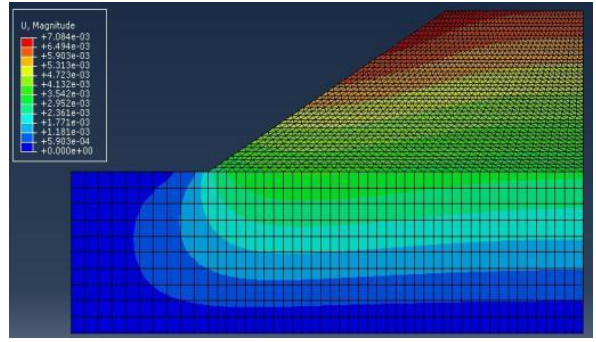

Fig.4 Total displacement field cloud map of slope without root system

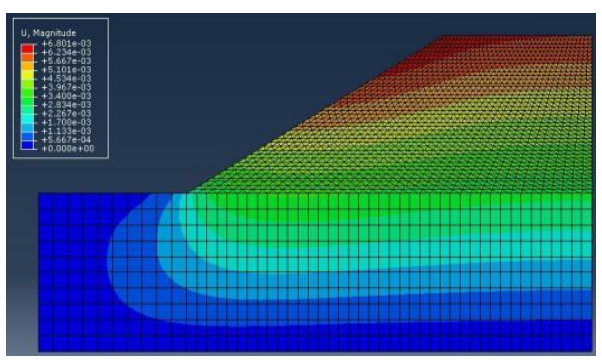

Fig.5 displacement field cloud map of slope with root system

Table 2 Surface displacement of slope (root distribution density of 10 plants $/ \mathrm{m}$ )

\begin{tabular}{|c|c|c|c|c|c|c|c|c|c|}
\hline Root length (mm) & \multicolumn{3}{|c|}{200} & \multicolumn{3}{c|}{300} & \multicolumn{3}{c|}{400} \\
\hline Branch amount & 3 & 5 & 7 & 3 & 5 & 7 & 3 & 5 & 7 \\
\hline $\begin{array}{c}\text { Horizontal displacement } \\
(\mathrm{mm})\end{array}$ & 1.252 & 1.241 & 1.232 & 1.246 & 1.230 & 1.218 & 1.237 & 1.219 & 1.202 \\
\hline $\begin{array}{c}\text { Vertical displacement } \\
(\mathrm{mm})\end{array}$ & 5.276 & 5.270 & 5.267 & 5.264 & 5.256 & 5.255 & 5.254 & 5.253 & 5.245 \\
\hline $\begin{array}{c}\text { Total displacement } \\
(\mathrm{mm})\end{array}$ & 5.374 & 5.368 & 5.353 & 5.361 & 5.351 & 5.349 & 5.355 & 5.347 & 5.337 \\
\hline
\end{tabular}

Table 3 Surface displacement of slope (root distribution density of 15 plants $/ \mathrm{m}$ )

\begin{tabular}{|c|c|c|c|c|c|c|c|c|c|}
\hline Root length (mm) & \multicolumn{3}{|c|}{200} & \multicolumn{3}{c|}{300} & \multicolumn{3}{c|}{400} \\
\hline Branch amount & 3 & 5 & 7 & 3 & 5 & 7 & 3 & 5 & 7 \\
\hline $\begin{array}{c}\text { Horizontal displacement } \\
(\mathrm{mm})\end{array}$ & 1.245 & 1.232 & 1.219 & 1.238 & 1.218 & 1.208 & 1.227 & 1.203 & 1.195 \\
\hline $\begin{array}{c}\text { Vertical displacement } \\
(\mathrm{mm})\end{array}$ & 5.271 & 5.263 & 5.257 & 5.261 & 5.252 & 5.193 & 5.249 & 5.239 & 5.144 \\
\hline $\begin{array}{c}\text { Total displacement } \\
(\mathrm{mm})\end{array}$ & 5.369 & 5.359 & 5.352 & 5.358 & 5.346 & 5.301 & 5.350 & 5.331 & 5.244 \\
\hline
\end{tabular}

To reflect the effect of length of fibrous on displacement of surface soil of slope more effectively, the variation curves of horizontal, vertical and total displacement were drawn out. When the arrangement density is 10 strains per meter, the results of displacement are showed in Fig.6, Fig.7, Fig.8 respectively; when the arrangement density is 15 strains per meter, the results of displacement are showed in Fig.9, Fig.10, Fig.11 respectively.

1) Arrangement density: 10 strains per meter

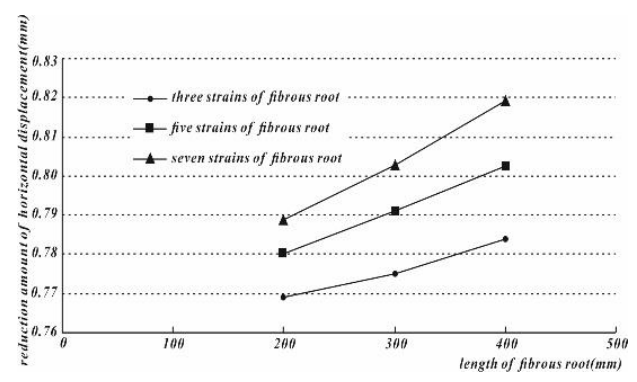

Fig.6 Curve graph of influence of fibrous root

length on horizontal displacement of slope surface soil

After fitting and analyzing the data above, it was 
indicated that it was more accurate to regress the relation between fibrous root length of bermuda grass root system and difference in horizontal displacement of slope surface by using linear fitting method. The fitting formulas are as follows:

Three strains of fibrous root:

$y=8 E^{-05} x+0.7535 \quad R^{2}=0.9868$

Five strains of fibrous root:

$y=0.0001 x+0.758 \quad R^{2}=1$

Seven strains of fibrous root:

$y=0.0002 x+0.7587 \quad R^{2}=0.9985$

Where: $x$-length of fibrous root $y$-difference of horizontal displacement $(\mathrm{mm})$

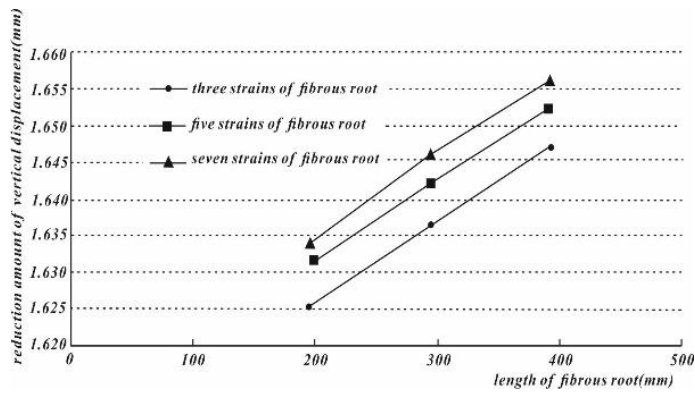

Fig.7 Curve graph of influence of fibrous root length on vertical displacement of slope surface soil

After fitting and analyzing the data above, it was indicated that it was more accurate to regress the relation between fibrous root length of bermuda grass root system and difference in vertical displacement of slope surface by using linear fitting method. The fitting formulas are as follows:

Three strains of fibrous root:

$y=0.0001 x+1.6033 \quad R^{2}=0.9973$

Five strains of fibrous root:

$y=0.0001 x+1.6102 \quad R^{2}=0.9992$

Seven strains of fibrous root:

$y=0.0001 x+1.6123 \quad R^{2}=0.9973$

Where: $x$-length of fibrous root $y$-difference of horizontal displacement $(\mathrm{mm})$

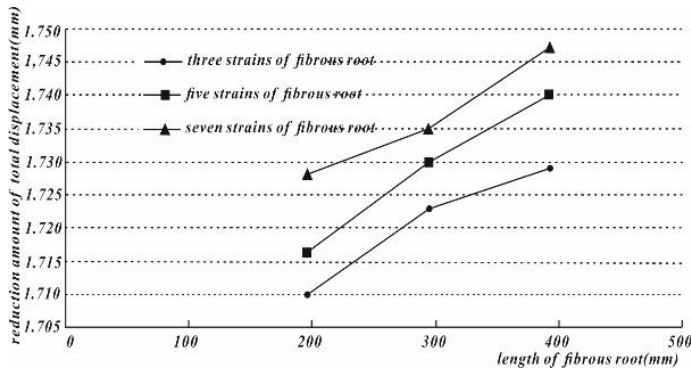

Fig.8 Curve graph of influence of fibrous root length

on total displacement of slope surface soil

After fitting and analyzing the data above, it was indicated that it was more accurate to regress the relation between fibrous root length of bermuda grass root system and difference in total displacement of slope surface by using linear fitting method. The fitting formulas are as follows:

Three strains of fibrous root:

$y=9 E^{-05} x+1.6922 \quad R^{2}=0.9867$

Five strains of fibrous root:

$y=0.0001 x+1.6927 \quad R^{2}=0.9908$

Seven strains of fibrous root:

$y=9 E^{-05} x+1.7082 \quad R^{2}=0.9874$

Where: $x$-length of fibrous root $y$-difference of horizontal displacement $(\mathrm{mm})$

2) Arrangement density; 15 strains per meter

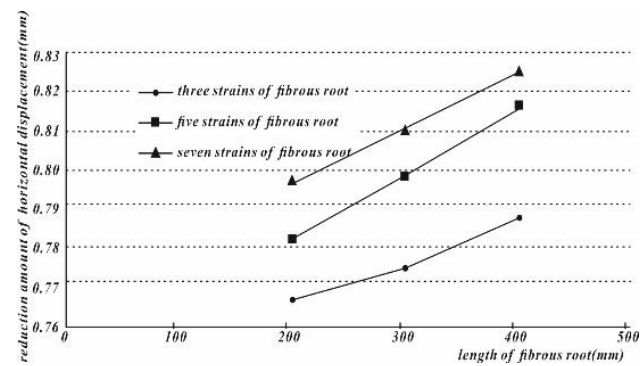

Fig.9 Curve graph of influence of fibrous root

length on horizontal displacement of slope surface soil

After fitting and analyzing the data above, it was indicated that it was more accurate to regress the relation between fibrous root length of bermuda grass root system and difference in horizontal displacement of slope surface by using linear fitting method. The fitting formulas are as follows:

Three strains of fibrous root:

$y=9 E^{-05} x+0.7573 \quad R^{2}=0.9838$

Five strains of fibrous root:

$y=0.0001 x+0.7598 \quad R^{2}=0.9996$

Seven strains of fibrous root:

$y=0.0001 x+0.7777 \quad R^{2}=0.9977$

Where: $x$-length of fibrous root $y$-difference of horizontal displacement $(\mathrm{mm})$ 


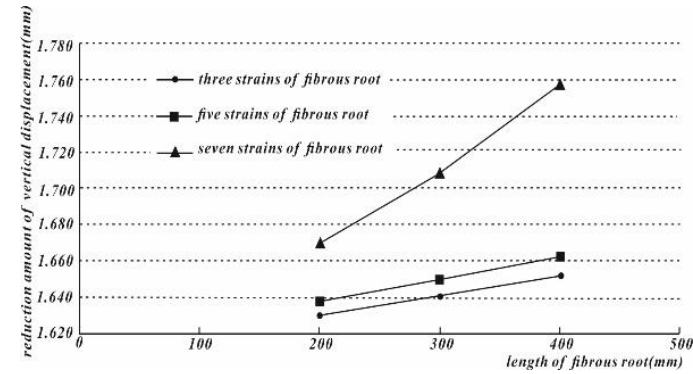

Fig.10 Curve graph of influence of fibrous root length on vertical displacement of slope surface soil

After fitting and analyzing the data above, it was indicated that it was more accurate to regress the relation between fibrous root length of bermuda grass root system and difference in vertical displacement of slope surface by using linear fitting method. The fitting formulas are as follows:

Three strains of fibrous root:

$y=0.0001 x+1.6077 \quad R^{2}=0.9973$

Five strains of fibrous root:

$y=0.0001 x+1.6137 \quad R^{2}=0.9977$

Seven strains of fibrous root:

$y=0.0004 x+1.5812 \quad R^{2}=0.9967$

Where: $x$-length of fibrous root $y$-difference of horizontal displacement $(\mathrm{mm})$

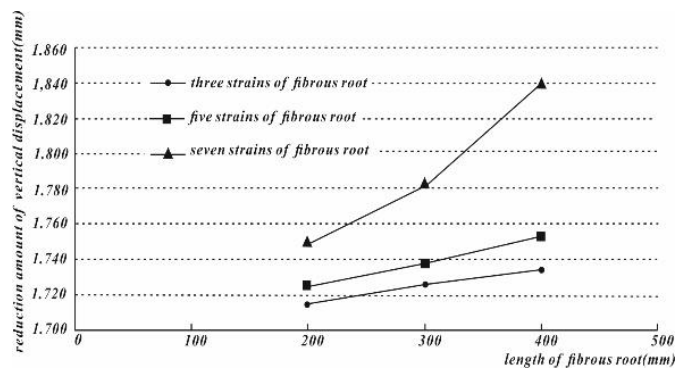

Fig.11 Curve graph of influence of fibrous root length on total displacement of slope surface soil

After fitting and analyzing the data above, it was indicated that it was more accurate to regress the relation between fibrous root length of bermuda grass root system and difference in total displacement of slope surface by using linear fitting method. The fitting formulas are as follows:

Three strains of fibrous root:

$y=1 E^{-04} x+1.6965 \quad R^{2}=0.9918$

Five strains of fibrous root:

$y=0.0001 x+1.6967 \quad R^{2}=0.9983$
Seven strains of fibrous root

$y=0.0005 x+1.656 \quad R^{2}=0.9868$

Where: $x$-length of fibrous root $y$-difference of horizontal displacement $(\mathrm{mm})$

In order to reflect the effect of arrangement density of root system on displacement of slope surface soil better, the differences in displacement between slope without root system and slope which was implanted with bermuda grass root system with 7 strains of fibrous root whose arrangement density of 10 strains per meter and 15 strains per meter were compared and analyzed. The variation curves of horizontal, vertical and total displacement were drawn out., showed in Fig.12, Fig.13, Fig.14.

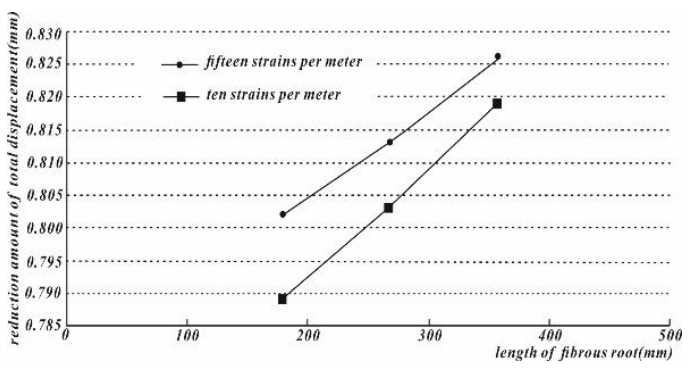

Fig.12 Curve graph of influence of fibrous root length on horizontal displacement of slope surface soil

After fitting and analyzing the data above, it was indicated that it was more accurate to regress the relation between fibrous root length of bermuda grass root system and difference in vertical displacement of slope surface by using linear fitting method. The fitting formulas are as follows:

Distribution density:10strains/meter

$y=0.0002 x+0.758 \quad R^{2}=0.9985$

Distribution density:15strains/meter

$y=0.0001 x+0.7777 \quad R^{2}=0.9977$

Where: $x$-length of fibrous root $y$-difference of horizontal displacement $(\mathrm{mm})$

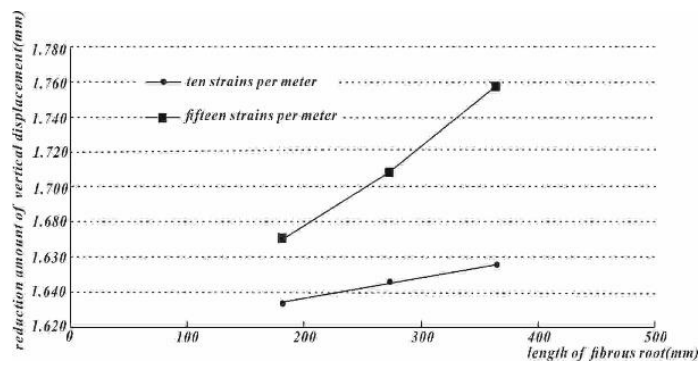

Fig.13 Curve graph of influence of fibrous root length 
on vertical displacement of slope surface soil

After fitting and analyzing the data above, it was indicated that it was more accurate to regress the relation between fibrous root length of bermuda grass root system and difference in vertical displacement of slope surface by using linear fitting method. The fitting formulas are as follows:

Distribution density:10strains/meter

$y=0.0001 x+1.6123 \quad R^{2}=0.9973$

Distribution density: 15 strains/meter

$y=0.0004+1.5812 \quad R^{2}=0.9987$

Where: $x$-length of fibrous root $y$-difference of horizontal displacement $(\mathrm{mm})$

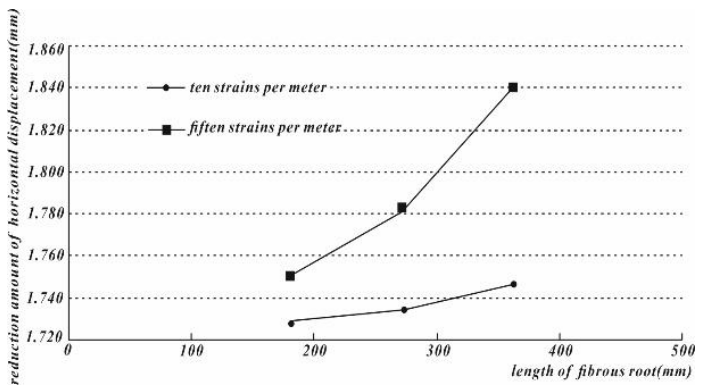

Fig.14 Curve graph of influence of fibrous root length

on total displacement of slope surface soil

After fitting and analyzing the data above, it was indicated that it was more accurate to regress the relation between fibrous root length of bermuda grass root system and difference in vertical displacement of slope surface by using linear fitting method. The fitting formulas are as follows:

Distribution density:10strains/meter

$y=9 E^{-05} x+1.7082 \quad R^{2}=0.9874$

Distribution density: 15 strains/mete

$y=0.0005 x+1.656 \quad R^{2}=0.9898$

Where: $x$-length of fibrous root $y$-difference of horizontal displacement $(\mathrm{mm})$

According to the data analysis, the amount, length and arrangement density of bermuda grass root system all have significant effects on the displacement of slope surface soil. According to the fitting formulas, for the slope within implanted plant whose arrangement density are 10 strains per meter and 15 strains per meter, the increase of difference in horizontal, vertical and total displacement with the increase of length of fibrous root is linear, more amount of fibrous root, longer length of fibrous root and more decrease of displacement. When the arrangement density is 15 strains per meter, the decrease of displacement of soil with the increase of length of fibrous root is more obvious. With the increase of amount of bermuda grass root, the performance of reducing slope sliding is easier to notice, the ability of anti-erosion become stronger, this reduces the probability of slope instability, strengthen the stability of slope.

\section{Conclusion}

By simulating the coaction between soil and bermuda grass root system with the finite element numerical calculation software, the effect of root system on the stability of soil were described quantitatively, the study result shows:

(1) The location of root system in the slope is related to the arrangement, amount of root system and length. The horizontal, vertical and total displacement of slope soil all decrease with the increase the of amount of fibrous root, among them, the decrease trend of vertical displacement and total displacement is larger than horizontal displacement. With the increase of length and amount of fibrous root, the effect of them on the displacement becomes more obvious.

(2) The length of bermuda grass root is short, mainly in the range of $39 \mathrm{~cm}$, the soil solidity performance of root system is performed within the sphere of influence of root system, but the amount of bermuda grass fibrous root id large and the elastic modulus of root system is much bigger than that of soil, so with the increase of amount of fibrous root and plant density, the cohesive force of soil increase, forming the reinforcement effect to soil. Especially in the condition of raining, the exist of root system changes the stress-strain state, the stiffness of slope surface soil becomes larger, this can effectively resist the erosion of slope surface by rain and constrain the displacement of soil, pretend the soil erosion. For the deep reinforcement of slope, other measures of reinforcement need to be taked into account to 
realize the performance of soil solidity.

(3) Because of spatial distribution and the complication of distribution pattern of plant root system, in this paper, the root morphology was simplified in the process of establishing model of root system, the actual root morphology is more complicate than that of simulation model. The problem of how to consider the actual distribution morphology of plant root system and real spatial features of slope soil is worth of being studied further.

\section{Acknowledgments}

This research was supported by Science and Technology Project Plan of Ministry of Housing and Urban-rural Development of the PRC [2016-K4-020], "Qing Lan Project" of universities in Jiangsu province, Jiangsu province "Six Talents Peak" project, Vocational College Teachers' Professional Leading High-level Research Project in Jiangsu province, Jiangsu Open University "The 13th Five-year Plan" project [17SSWZ-Y-024].

\section{References}

[1] Kundu, Jagadish, et al. "Deterministic and Probabilistic Stability Analysis of Soil Slope - A Case Study."Journal of the Geological Society of India 2018: 91(4):418-424.

[2]. Hayati, Elyas, et al. "Soil water dynamics under different forest vegetation cover: Implications for hillslope stability."Earth Surface Processes \& Landforms 2018:1-2.

[3]. Wieder, Wendy L., and S. A. Shoop. "State of the knowledge of vegetation impact on soil strength and trafficability. "Journal of Terramechanics2018:78:1-14. [4]. Song, Shuangshuang, et al. "Effects of Root Properties and Branching Characteristics on Soil Reinforcement in the Jinyun Mountain, China."Current Science 2018:114(6):1250.

[5]. Liang, T., et al. "Scaling of the reinforcement of soil slopes by living plants in a geotechnical centrifuge."Ecological Engineering 2017:109.

[6]. Li, Yunpeng, Y. Wang, and S. Song. "Effects of Root Architecture Characteristics on Soil
Reinforcement in Undisturbed Soil."Current Science 2017:113(10):1993-2003.

[7] Cislaghi, Alessio, E. A. Chiaradia, and G. B. Bischetti. "Including root reinforcement variability in a probabilistic 3D stability model."Earth Surface Processes \& Landforms 2017:(42).

[8] Boldrin, D., A. K. Leung, and A. G. Bengough. "Correlating hydrologic reinforcement of vegetated soil with plant traits during establishment of woody perennials."Plant \& Soil 2017: 416(1-2):437-451.

[9] Ye, Chao, et al. "The effect of Bahiagrass roots on soil erosion resistance of Aquults in subtropical China."Geomorphology2017:(285):82-93.

[10] $\mathrm{Hu}$ ping, Song xiuguang, and $\mathrm{Wu}$ denggao. Experimental study of root-solid effect of grass planting and slope protection on highway slope. Geotechnical Mechanics 2008: 29(2):442-444.

[11] Zhou junping et al. Research progress on the effects of vegetation on slope stability. Highway 2013: 33(6):9-15.

[12] Thompson, A. G., and E. Villaescusa. Case Studies of Rock Reinforcement Components and Systems Testing. Rock Mechanics \& Rock Engineering 2014:1589-1602.

[13]Yunyan, Z., Jianping, C., Xiaomei, W., Research progress and prospect of the mechanism of soil protection in plant root system $[\mathrm{J}]$. Journal of ecological environment,2012:21(6): 1171-1177.

[14]. Han, L., Tingning, Z., Xianfeng, P., et al. Experimental study on the effect of soil and water conservation on highway slopes $[\mathrm{J}]$. Journal of agricultural engineering,2013:29(5):63-70.

[15]. Huatan, L., Xiagao, H., Yujiao Z.,, et al. Research progress on the mechanism of strengthening soil shear strength in plant roots [J]. People's Yellow River,2014: 36(8):97-100.

[16]. Zhixin, S., Jie, S., Hancheng C., et al. Mechanical principles of the reinforcement of the slope of herbs $[\mathrm{J}]$. Civil architecture and environmenta engineering,2010:32(2):30-33.

[17]. Zendda, C., Hongbin, X., Chunxiao, Z., et al. Effects of root distribution on shear strength of root soil complex $[\mathrm{J}]$. Journal of central south university of forestry and technology,2016:36(8):130-135.

[18]Zengning,D., Yujiao,Z., Changyi,L.,et al.Soil 
displacement and earth pressure characteristics of slopes in a cold and arid region under rainfall
simulation[J].Hydrogeology

\&

engineering 